\title{
La répartition de l'énergie dans les sections de mesure de conduites de pompage et son influence sur l'exactitude d'un rendement mesuré par la méthode thermodynamique
}

PAR

\author{
Joseph Brun \\ Docteur en Sciences Techniques
}

\section{Introduction}

La mesure thermique du rendement, dans les machines hydrauliques, consiste à déterminer la quantité d'énergie introduite ou emportée par le fluide en écoulement, ainsi que la quote-part de cette énergie récupérable à entropie constante. A cet effet, on détermine dans les sections de mesure, en un ou en plusieurs endroits, la pression totale et la température, ainsi que la pression statique régnant dans ces sections.

Si l'on procède à la mesure en plusieurs endroits des sections d'écoulement, procédé recommandé, mais souvent sujet à des difficultés, ou même impossible à appliquer, on peut déterminer le débit d'énergie moyen. Dans le cas de la mesure en un seul endroit, en revanche, celle-ci ne porte que sur le débit d'énergie affecté au filet liquide correspondant, et qui peut différer du débit d'énergie moyen. Cet écart a son influence sur l'exactitude des résultats obtenus avec un seul point de mesure.

$\left.{ }^{*}\right)$ Texte abrégé de la thèse de doctorat de l'auteur, acceptée sous le même titre en 1975 par la section des sciences de l'ingénieur à l'Ecole polytechnique fédérale (EPF) de Zurich. Ce sont Messieurs H. Gerber, prof., et P.U. Weber, chargé de cours, à l'Institut pour Machines et Installations hydrauliques, à l'EPF Zurich, qui ont incité l'auteur à entreprendre cette étude. Les investigations ont été faites en bonne partie dans les laboratoires de la Maison Sulzer Frères, Société Anonyme, à Winterthur ; c'est aussi cette maison qui a mis gracieusement à disposition les dispositifs de mesure et les pompes d'essai. Une autre partie des investigations a été effectuée sur une pompe d'accumulation, dans la centrale de Veytaux des Forces Motrices de l'Hongrin, à l'occasion des essais de réception qui y ont été faits sous la direction de Monsieur Th. Bovet, professeur à l'EPF Lausanne. L'auteur remercie ici vivement, pour leur appui, Messieurs H. Gerber, prof., B. Chaix, dr. ing., prof. (tous deux à l'EPF Zurich), ainsi que P.U. Weber, chargé de cours.

\section{Enoncé du problème}

Les mesures faites sur des turbines Pelton par les promoteurs de la méthode thermodynamique pour la mesure du rendement, avaient montré qu'il suffit de déterminer les grandeurs caractéristiques de l'état de l'eau en un seul endroit, c'est-à-dire dans la conduite forcée [1] [2] [3] [4]. Il n'y a là rien d'étonnant car les turbines Pelton travaillant sous des chutes de quelques centaines de mètres, leur conduite forcée est longue en conséquence. Mais on sait aussi par expérience qu'en présence d'un organe de fermeture, à condition qu'il ne s'agisse pas d'une vanne sphérique, il faut se garder de procéder à une mesure à peu de distance en aval de cet organe. S'il est vrai qu'après un objet solide, placé dans l'écoulement, l'énergie totale se maintient inchangée sur la longueur de celui-ci, l'équilibre de pression et de température ne s'établit cependant pas au même endroit. C'est pour cette raison qu'on déplacera un point de mesure à l'entrée de la machine, après l'organe de fermeture dans le sens de l'écoulement, aussi loin que possible à l'aval de cet organe, c'est-à-dire immédiatement avant la turbine. La situation est moins claire à la sortie d'une turbine Pelton, d'une turbine à contrepression, ou d'une pompe, car, dans ces machines, les sources de pertes (soit les aubes) sont réparties par points ou par surfaces partielles, ce qui conduit à une répartition correspondante, après la machine de la température, et donc de l'énergie. Des constatations faites dans ce domaine sur des turbines Pelton ont incité les spécialistes de ces mesures à poser certaines exigences portant sur l'observation d'un "parcours de mélange suffisant" ; l'Electricité de France impose, dans tous les cas, que toute mesure dans le canal de fuite de turbines Pelton soit complétée par celle de la répartition de la température. 
Le but de la présente investigation est de déterminer. dans le cas des pompes, les écarts de la pression et de la température, par rapport aux valeurs moyennes, d'après l'emplacement de l'endroit de mesure dans la section considérée, à l'aval de la machine, la longueur du parcours de mélange (entre la sortie de la roue et la section de mesure), le point de fonctionnement de la pompe, et enfin suivant la vitesse spécifique de celle-ci et ses particularités de construction. Les valeurs mesurées pour la pression totale, la pression statique et la température, permettent de calculer la répartition des hauteurs représentatives d'énergies (hauteur de refoulement et hauteur énergétique totale) nécessaires à la détermination du rendement par calcul thermodynamique, de même que leur influence sur l'exactitude du résultat obtenu en fin de compte pour le rendement.

\section{Bases théoriques}

Par sa définition même [5], le rendement interne d'une machine en écoulement continu est égal au rapport de la puissance fournie à la pompe, exempte de pertes, à la puissance effective fournie par la roue, ou de la puissance fournie par la roue à celle de la machine sans pertes dans lc cas de la turbine. On peut, dans le calcul, remplacer la puissance par le travail rapporté à l'unité de masse, introduit dans le fluide actif ou fourni par lui.

Ce travail s'établit comme la différence $\Delta h$ d'enthalpie totale, entre l'entrée et la sortie, et la chaleur échangée entre le fluide de travail et son entourage, ici aussi considérée entre l'entrée et la sortie. L'enthalpie totale $h$ [6] [7] peut se calculer, à partir de l'énergie interne $u$, de la pression $p$, du volume spécifique $v$, de la vitesse $c$. de la cote d'implantation $z$ et enfin de la constante de gravitation $g$, de la façon suivante :

$$
h=u+p \cdot v+\frac{c^{2}}{2}+g \cdot z
$$

Dans une machine travaillant sans pertes, la puissance apportée au fluide actif, ou foumie par ce fluide, est égalle à la différence $\Delta h_{s}$ d'enthalpie totale, entre l'entrée et la sortie, déterminée dans l'hypothèse d'une énergie constante.

L'enthalpie mesurée en un point $x$ d'une section diffère de l'enthalpie totale de densité moyenne, portant sur toute la section, et qu'on aura déterminée en tenant compte de la répartition de la densité $\rho$, par les différences $\delta h_{x}$ et $\delta h_{s . x}$ :

$$
\begin{aligned}
\delta h_{x}= & \left(u+p \cdot v+\frac{c^{2}}{2}+g \cdot z\right)_{x}- \\
& -\frac{\int_{F}\left(u+p \cdot v+\frac{c^{2}}{2}+g \cdot z\right) \cdot \rho \cdot c \cdot d F}{\int_{F} \rho \cdot c \cdot d F} \\
\delta h_{s x}= & \left(p \cdot v+\frac{c^{2}}{2}+g \cdot z\right)_{x}- \\
& -\frac{\int_{F}\left(p \cdot v+\frac{c^{2}}{2}+g \cdot z\right) \cdot p \cdot c \cdot d F}{\int_{F} p \cdot c \cdot d F}
\end{aligned}
$$

On peut en déduire, rapportées aux différences d'énergie $\Delta h$ et $\Delta h_{s}$, les différences relatives $\psi h_{x}$ et $\psi h_{s x}$ :

$$
\Psi h_{x}=\frac{\delta h_{x}}{\Delta h} ; \Psi h_{s x}=\frac{\delta h_{s x}}{\Delta h_{s}}
$$

Si, au cours de la détermination du rendement, on ne mesure les valeurs caractéristiques de l'état qu'en un seul point de la section, les grandeurs $\psi h_{x}$ et $\psi h_{s x}$ affectées à ce point de mesure grèvent ensuite d'erreurs systématiques toute l'interprétation des résultats.

\section{Essais tendant à déterminer la répar- tition de l'énergie dans les sections de sorties de pompes}

\section{But et destination de ces essais}

Les mesures faites sur trois machines-modèles, et sur une pompe en exécution industrielle, avaient pour but de déterminer la répartition de l'énergie en différents points de la section de sortie, et sous diverses conditions de marche.

Dans le cas des machines-modèles, les sections de mesure ont été examinées chacune au moyen de 48 points de mesure, répartis uniformément sur la périphérie de quatre cercles concentriques et affectés à des secteurs circulaires de même surface. Dans l'exécution industrielle, chaque section examinée a été partagée en 20 points de mesure répartis sur quatre rayons et affectés à des secteurs de même surface.

L'énergie interne obtenue, par la mesure au point $x$, par rapport à un point de référence, est désignée par $\Delta u$. D'autre part, on peut admettre que la valeur $p . v+g . z$ reste constante sur la section tout entière, et la valeur admise, dans le calcul, pour la densité est, à titre approché, la grandeur $\rho$ affectée au fluide dans l'axe du tuyau. Compte tenu de la condition d'après laquelle les $\mathrm{N}$ points de mesure ( 48 dans les machines-modèles, et 20 dans l'exécution en grandeur d'usine) sont affectés à des secteurs de surface égale, on obtient les relations simplifiées suivantes:

$$
\begin{aligned}
& \delta h_{x}=\left(\Delta u+\frac{c^{2}}{2}\right) x-\frac{\sum_{1}^{N} \xi\left(\Delta u+\frac{c^{2}}{2}\right)_{\xi} \cdot c_{\xi}}{\sum_{i}^{N} \xi c_{\xi}} \\
& \delta h_{s i x}=\left(\frac{c^{2}}{2}\right) x-\frac{\sum_{1}^{N} \xi\left(\frac{c^{2}}{2}\right)_{\xi} \cdot c_{\xi}}{\sum_{1}^{N} \xi c_{\xi}}
\end{aligned}
$$

Les grandeurs ainsi obtenues permettent de déterminer les valeurs de $\psi h_{x}$ et $\psi h_{s x}$. Les essais poursuivis sur ces bases avaient pour but de determiner dans quelle mesure ces valeurs $\psi h_{x}$ et $\psi h_{s x}$ sont influencées par le point de régime, la position de l'endroit de la mesure, la longueur du parcours de compensation entre la section de mesure et la sortie de la roue, ainsi que par les caractéristiques de construction de la machine. 
Il reste encore à désigner, dans les considérations suivantes, la grandeur $\delta h$ comme étant l'écart de l'énergie totale, et la grandeur $\delta h_{s}$ comme celui de l'énergie hydraulique. De manière analogue, la valeur $\Delta h$ désignera l'énergie totale, et la grandeur $\Delta h_{s}$ l'énergie hydraulique transformée dans la machine.

\section{Machines essayées}

Les machines examinées dans cette étude sont des pompes à haute pression à une ouie d'entrée, avec appareil directeur et bâche-spirale de sortie : le tableau ci-dessous en donne les caractéristiques de service principales

\begin{tabular}{|l|c|c|c|c|}
\hline \multicolumn{1}{|c|}{ Machine d'essai } & A & B & C & D \\
\hline Nombre de tours spécifique $n_{s} *$ & 158 & 154 & 139 & 120 \\
Nombre d'étages $z$ & 3 & 4 & 1 & 5 \\
Diamètre extérieur de la roue & & & & \\
à aubes $D_{L a}(\mathrm{~m})$ & 0,381 & 0,381 & 0,400 & 1,850 \\
Débit refoulé $Q_{f}\left(\mathrm{~m}^{3} / \mathrm{s}\right)$ & 0,185 & 0,193 & 0,340 & 6,500 \\
$\begin{array}{l}\text { Hauteur de refoulement } \\
H_{f}(\mathrm{~m} \text { d'eaul) }\end{array}$ & 110 & 155 & 166 & 838 \\
Vitesse de rotation $n(\mathrm{t} / \mathrm{mn})$ & 1500 & 1500 & 3000 & 600 \\
Puissance absorbée $P_{w}(\mathrm{~kW})$ & 229 & 331 & 638 & 59200 \\
Rendement $\eta_{p}$ & 0,874 & 0,886 & 0,870 & 0,901 \\
\hline
\end{tabular}

$$
\left.{ }^{*}\right) n_{s}=3,65 \cdot n \cdot \frac{\sqrt{Q_{f}}}{H_{f}^{3 / 4}}
$$

\section{Dispositif d'essais}

Le dispositif d'essais comprend une sonde balayant toute la section de mesure sur un diamètre, et conformée soit en jauge cylindrique à pression dynamique, soit aussi en tube de soutirage cylindrique ; ce dispositif est complété par un appareil de fixation, ainsi que par les instruments de mesure de la pression et par un instrument pour la mesure différentielle de la température.

La sonde transversale utilisée pour les mesures sur les machines-modeles comprend quatre forages pour mesure de la pression dynamique, répartis sur l'une de ses branches, tandis que sur l'autre branche se trouvent, répartis de façon identique, quatre forages de prise servant aux mesures de température; au milieu de ces branches, une ouverture de prise peut servir à la mesure de la pression ou à celle de la température de référence. Des robinets à boisseau rotatif permettent de mettre les divers forages à volonté en communication avec des chambres internes de mesure de pression et de température.

La sonde transversale est insérée dans un tronçon de tube susceptible de toumer librement autour de son axe ; ce tube rotatif peut être fixé directement, par bride. sur la sortie de la pompe, ou par l'intermédiaire de tubes d'entretoise, ce qui permet de procéder à la mesure à des distances de $0,35,1,10$ et $1,85 \mathrm{~m}$ à partir de la sortie de la spirale (sections de mesure A, B et C).

La pression statique agissant sur la paroi du tube est mesurée au moyen d'un manomètre à piston, tandis que la pression dynamique qui s'établit entre la sonde de mesure et la paroi du tube est déterminée par un manomètre différentiel. La différence de température qui intervient entre celle du point de mesure et celle du point de référence s'obtient à l'aide d'un dispositif formé de thermometres à résistances, d'un pont à courant continu et d'un galvanometre.

Pour les essais faits sur la pompe d'accumulation D, les conduites d'amenés et de sortie de celle-ci furent pourvues chacune de deux sondes transversales disposées à $45^{\circ}$ sur l'horizontale. Chacune d'elles est pourvue de dix forages, répartis sur le pourtour et servant d'ouvertures pour mesures de pression et prises d'eau; ces ouvertures peuvent être mises en communication à volonté, par un robinet à boisseau, avec une chambre intérieure. Les mesures de pression et de température ont eté faites à l'aide d'instruments adéquats disposées à l'extérieur des conduites [8].

La figure 1 représente la disposition dessais appliquée a la pompe-modele $A$, tandis que la disposition utilisée pour la pompe d'accumulation $D$ est visible sur la figure 2 .

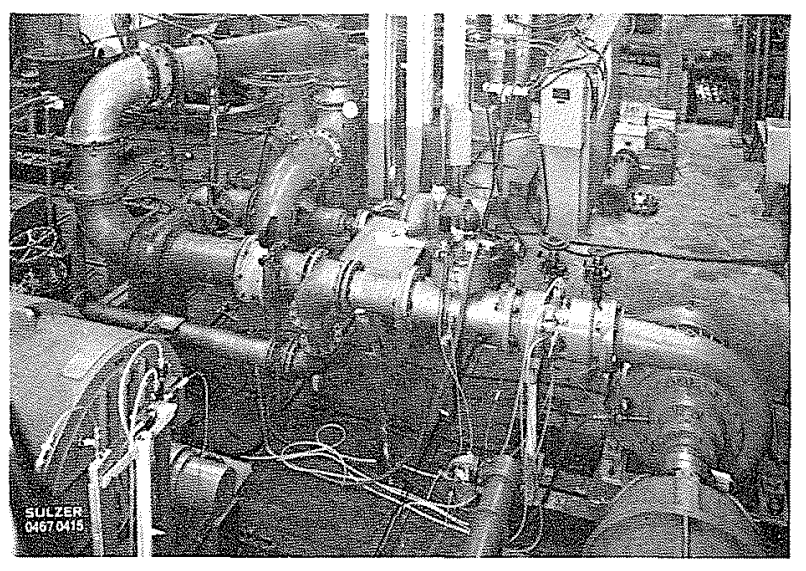

1/ Disposition d'essai avec la pompe-modèle A

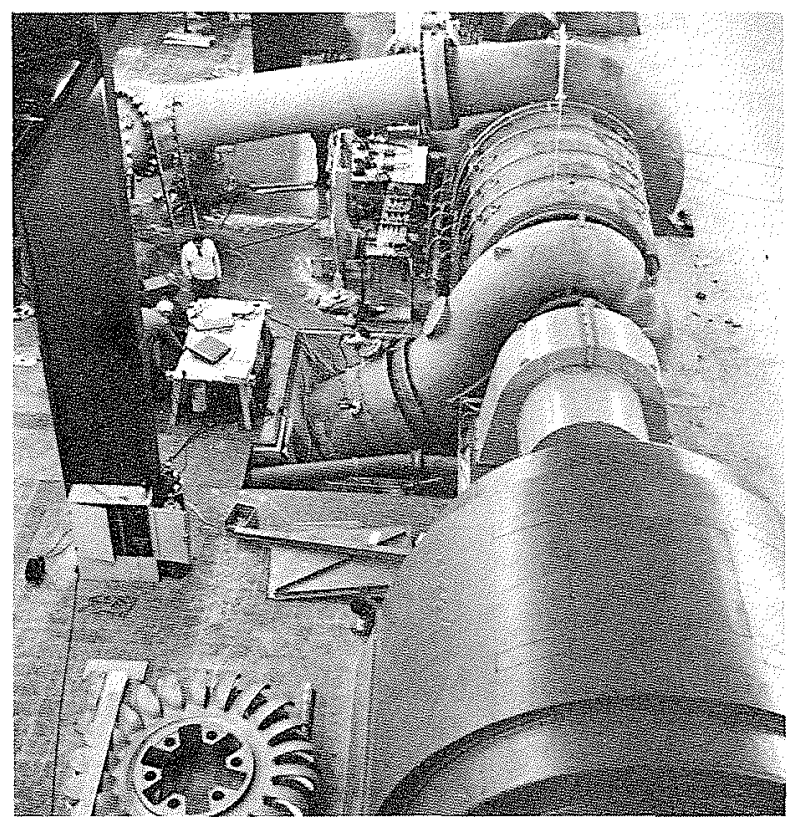

2/Disposition d'essai avec la pompe d'accumulation D 
Programme et exécution des essais

La série des essais a porté sur les mesures suivantes :

- Mesure de la répartition de l'énergie dans les sections de mesure $\mathrm{A}$ et $\mathrm{B}$ de la pompe-modèle $\mathrm{A}$, à pleine charge comme au débit partiel de $80 \%$ et en surcharge à $120 \%$ du régime nominal.

- Mesure de la répartition de l'énergie dans les sections de mesure $\mathrm{A}, \mathrm{B}$ et $\mathrm{C}$ des pompes-modèles $\mathrm{B}$ et $\mathrm{C}$, à pleine charge comme à charges partielles de $60 \%$ et $80 \%$ et en surcharge à $120 \%$ du débit refoulé nominal.

- Mesure de la répartition de l'énergie dans une section de mesure de la pompe d'accumulation $D$, au point de marche à débit optimum.

\section{Interprétation des résultats des mesures}

La vitesse d'écoulement de l'eau $c_{x}$ en $\mathrm{m} / \mathrm{s}$ est calculée, à partir de la pression dynamique $\Delta H$, en mètres d'eau, agissant au point $x$ et déterminée par l'indication du manomètre différentiel, ainsi que du coefficient $k$ de la sonde dynamique (posé égal à 1), de la manière suivante

$$
c_{x}=4,43 \cdot k \cdot \sqrt{\Delta H}
$$

La vitesse d'écoulement moyenne de l'eau $c_{m}$ est égale à la moyenne de toutes les valeurs $c_{x}$ obtenues dans la section de mesure correspondante.

Pour l'énergie hydraulique, on détermine, d'après l'équation posée ci-dessous, la différence relative $\psi h_{s x}$ entre la valeur mesurée dans le filet d'écoulement $x$, et la valeur moyenne dans la section de mesure, obtenue à partir des pressions dynamiques $\Delta H$ en $\mathrm{m}$ d'eau, les vitesses d'écoulement $c$ en $\mathrm{m} / \mathrm{s}$ et la hauteur de refoulement $H_{f}$ en m d'eau :

$$
\Psi h_{s, x}=\frac{\Delta H_{x}}{H_{f}}-\frac{1}{N} \frac{\sum_{1}^{N} \xi \Delta H_{\xi} \cdot \frac{c_{\xi}}{c_{m}}}{H_{f}}
$$

Pour l'énergie totale, on détermine de manière analogue, d'après l'équation ci-dessous, la différence relative $\psi h_{x}$. entre la valeur mesurée dans le filet d'écoulement $x$ et la valeur moyenne dans la section de mesure, obtenue à partir des pressions dynamiques $\Delta H$ en $\mathrm{m}$ d'eau, les différences d'énergie $\Delta u$ en $m$ d'eau, les vitesses d'écoulement $c$ en $\mathrm{m} / \mathrm{s}$ et la hauteur d'énergie dynamique de la roue $H_{u}$ en m d'eau :

$$
\Psi h_{x}=\frac{(\Delta u+\Delta H)_{x}}{H_{u}}-\frac{1}{N} \frac{\sum_{1}^{N} \xi(\Delta u+\Delta H)_{\xi} \cdot \frac{c_{\xi}}{c_{m}}}{H_{u}}
$$

Le niveau d'énergie de la roue $H_{u}$ est donné par la hauteur de refoulement $H_{f}$ et le rendement interne $\eta_{i}$, soit par la puissance absorbée par la roue et le débit refoulé $Q_{f}$. La différence d'énergie $\Delta u$ est déterminée d'après la lecture au galvanomètre et par les constantes d'instruments 17] obtenues par l'étalonnage de ces derniers.

\section{Evaluation des erreurs}

Lors de la détermination de la répartition de l'énergie hydraulique, les écarts occasionnels relatifs, par rapport à la valeur locale moyenne, peuvent causer une erreur inférieure à $\pm 0,2 \%$, pour une inexactitude de $\pm 1,0 \mathrm{~mm}$ dans la lecture du manomètre. Lors de la détermination de l'énergie totale, ces écarts occasionnels atteindront également moins de $0,2 \%$ pour la même inexactitude de lecture au manomètre, celle de la lecture au galvanomètre étant de $\pm 0,2 \mathrm{~mm}$, et le courant au pont de mesure pouvant varier de $\pm 0,1 \mathrm{~mA}$. Quant aux autres grandeurs de mesure, telles que le débit refoulé, la hauteur de refoulement et la vitesse de rotation, les exactitudes de mesure sont admises selon les règles VDI [9] [10]

\section{Résultats des essais}

\section{Répartition de l'énergie}

La répartition de l'énergie hydraulique est sujette aux écarts les plus importants dans les sections proches de la sortie de la pompe, à proximité de la paroi de la conduite, en régime de surcharge et enfin dans les pompes à faible nombre d'étages. C'est pour la répartition de l'énergie totale, au voisinage de la paroi de la conduite, ainsi qu'en régime de surcharge et de charge partielle, de mème enfin que dans les pompes à faible nombre d'étages, que l'on constate les plus grands écarts. Les figures 3 et 4 montrent, à titre d'exemple, les résultats obtenus sur la pompe-modèle B.

En considérant les répartitions d'énergie sous une forme empirique [11], on peut en calculer la dispersion de la manière suivante :

$$
s \Psi h_{s}=\sqrt{\frac{\sum_{1}^{N} i \Psi h s^{2} i}{N-1}} ; \quad s \Psi h=\sqrt{\frac{\sum_{1}^{N} i \Psi h^{2}{ }_{i}}{N-1}}
$$

Influence du point de marche, du parcours de compensation et du genre de machine sur la répartition de l'énergie

Les figures 5 à 7 font ressortir la dispersion de la répartition de l'énergie constatée sur les pompes-modèles, suivant le débit refoulé relatif avec, en paramètre, la section de mesure. La dispersion de l'énergie hydraulique diminue à mesure que le parcours de compensation est plus long ; quant à la dispersion de l'énergie totale, aucune influence correspondante n'a pu être constatée dans tout le domaine des parcours de compensation sur lequel a porté notre étude. La figure 8 fait ressortir l'influence du nombre d'étages de la pompe sur la dispersion de la répartition d'énergie au débit de marche optimum : la dispersion diminue suivant une hyperbole à mesure que le nombre d'étages augmente. Si l'on introduit dans le calcul, au lieu de la hauteur de refoulement et celle de l'énergie à la roue de la pompe, les valeurs correspondantes d'un étage isolé, on constate, comme le montre la figure 9, que le nombre d'étages n'a plus qu'une influence minime sur la dispersion. La figure 10 montre d'autre part qu'on ne peut déceler aucune influence du nombre de tours spécifique. 

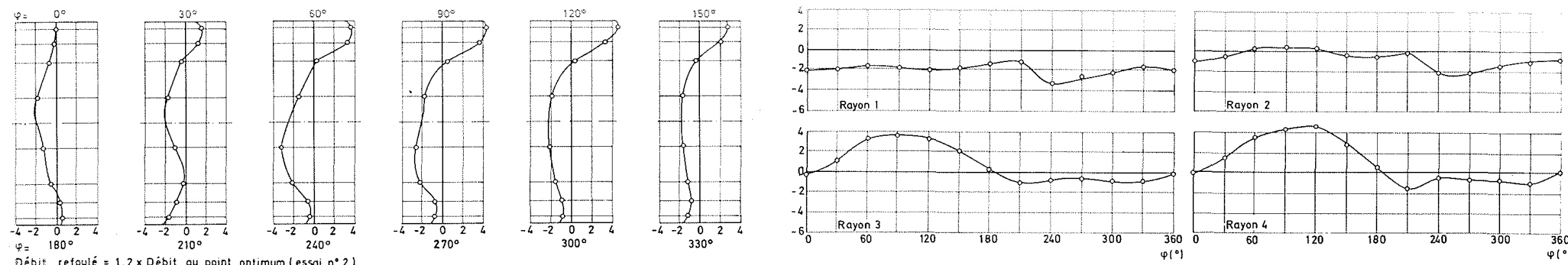

$\frac{1}{-4-2024}$

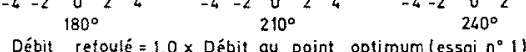
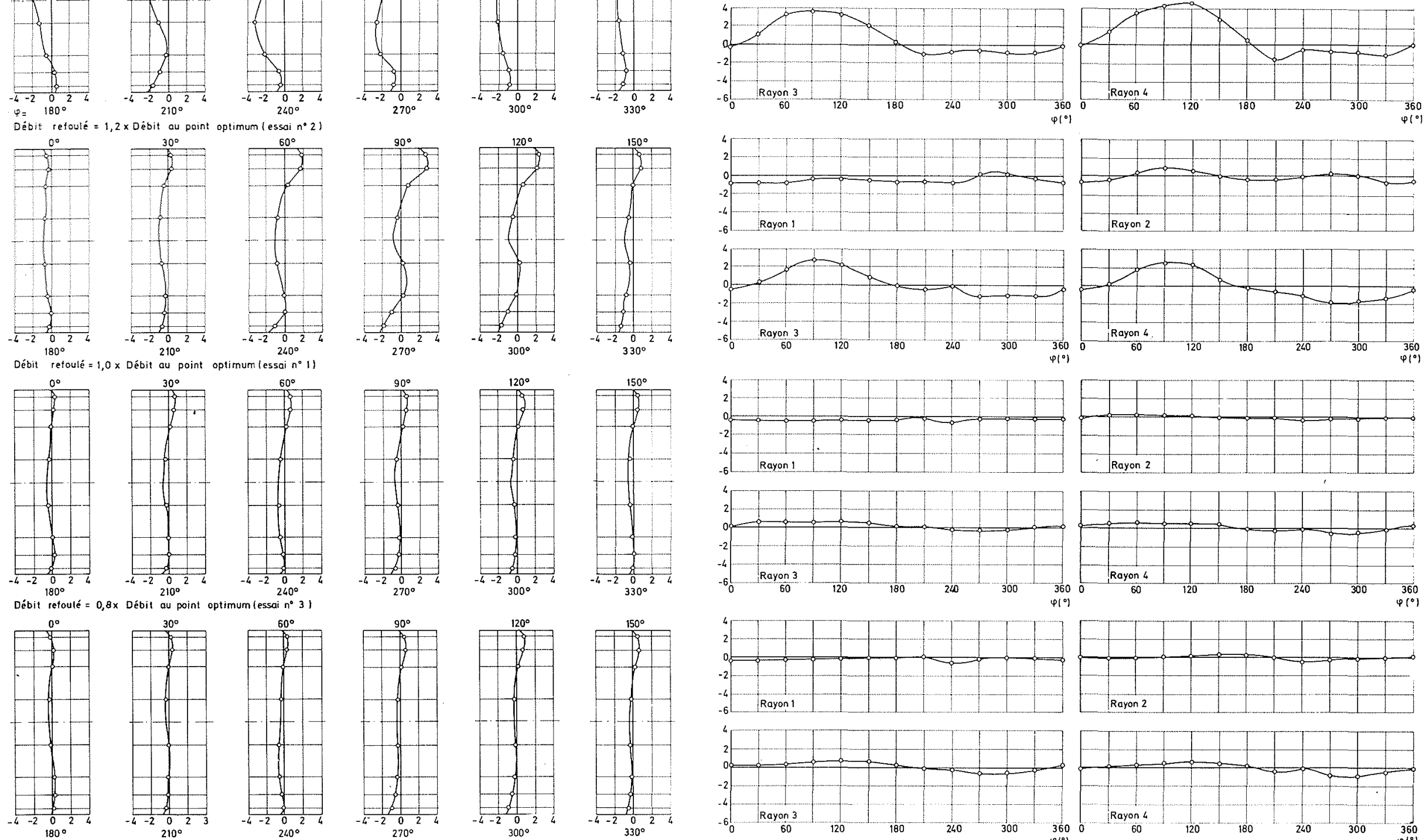

Débit retoulé $=0,6 \times$ Débit au point optimum (essai $n^{\circ} 4$ )
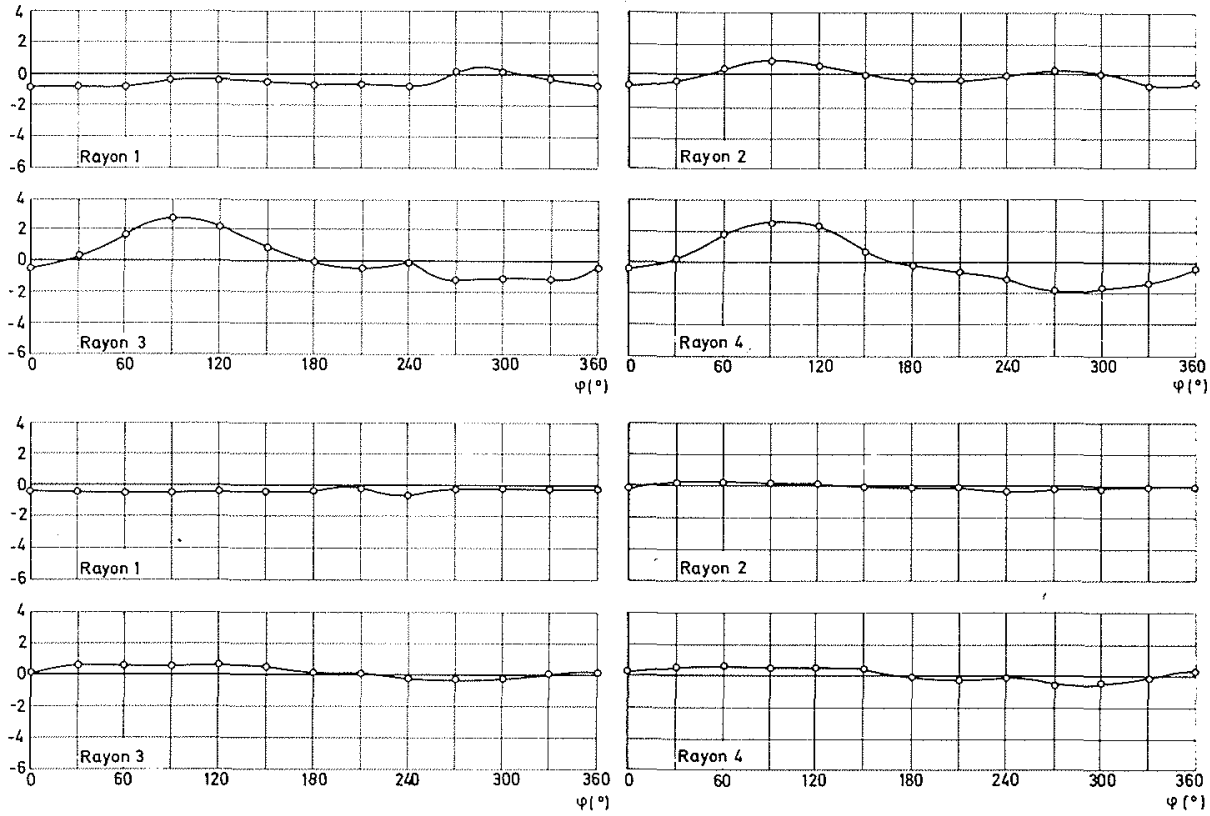

3/ Ecarts de l'énergie hydraulique, par rapport à la valeur moyenne, dans la section de mesure $A$ de la pompe-modèle $B$ en \%o 

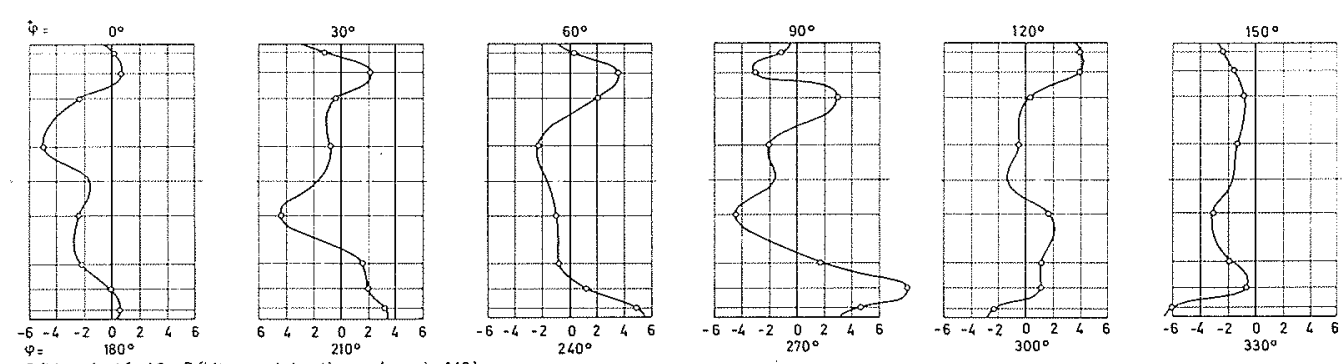

$\int_{-2}^{2} \int_{\text {Rayon } 1}^{2}$

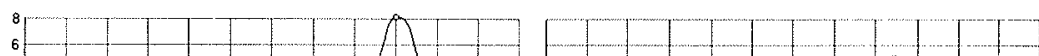
$\underset{-2}{\operatorname{Ray0} 30} \underset{120}{60}$
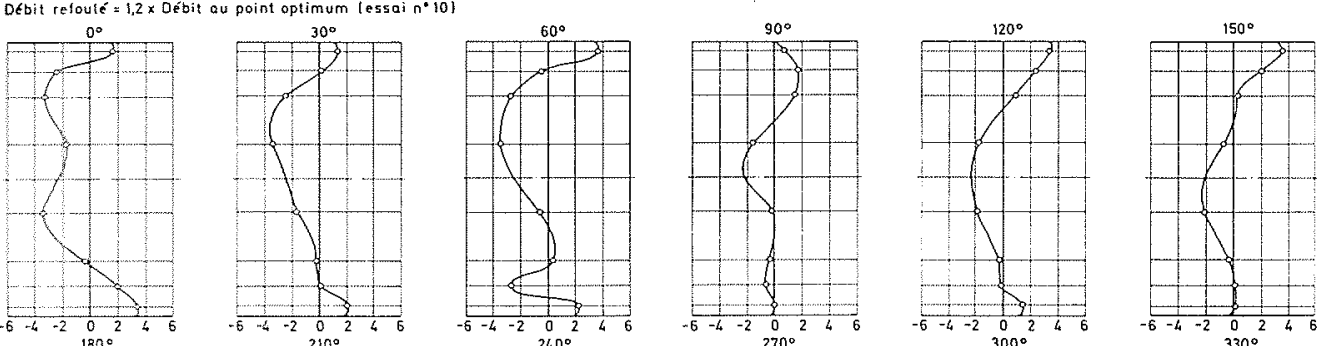

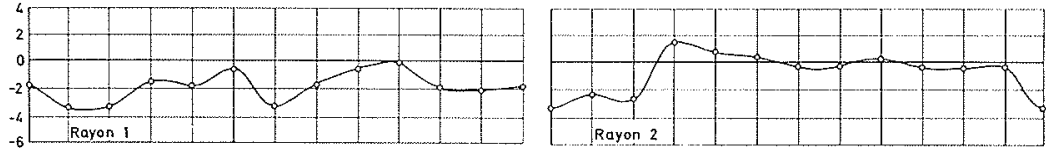

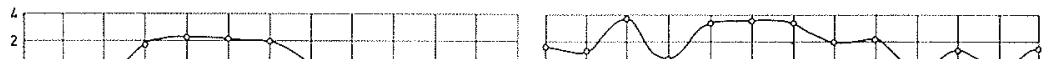
$\underset{\substack{\text { Royon } 3 \\ 60}}{120}$ Débit retoulé $=1.0 \times$ Débit au point optimum lessai $n^{\circ}$ )
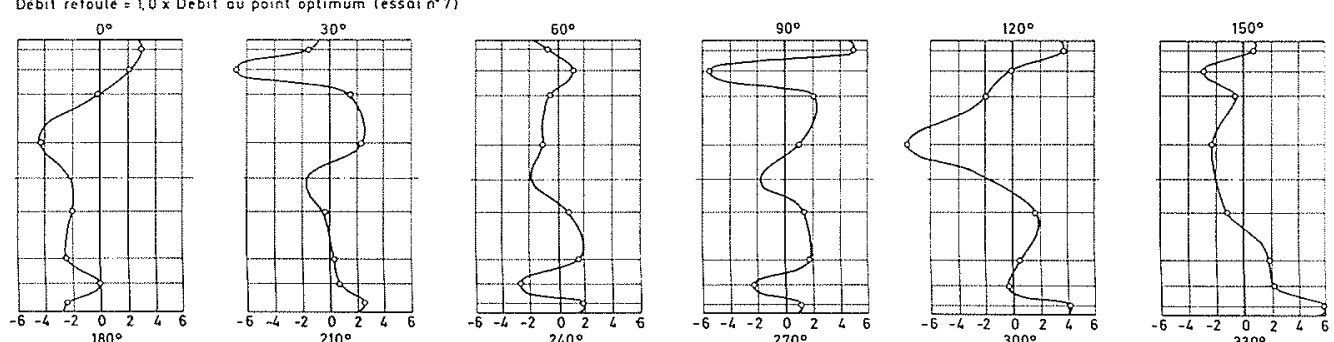

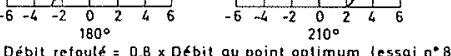
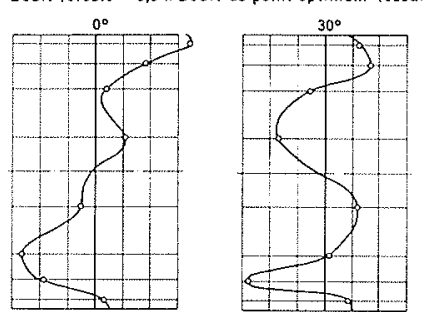

Debil refoule $=0,6$ Debit au point optimum lessai n.9.
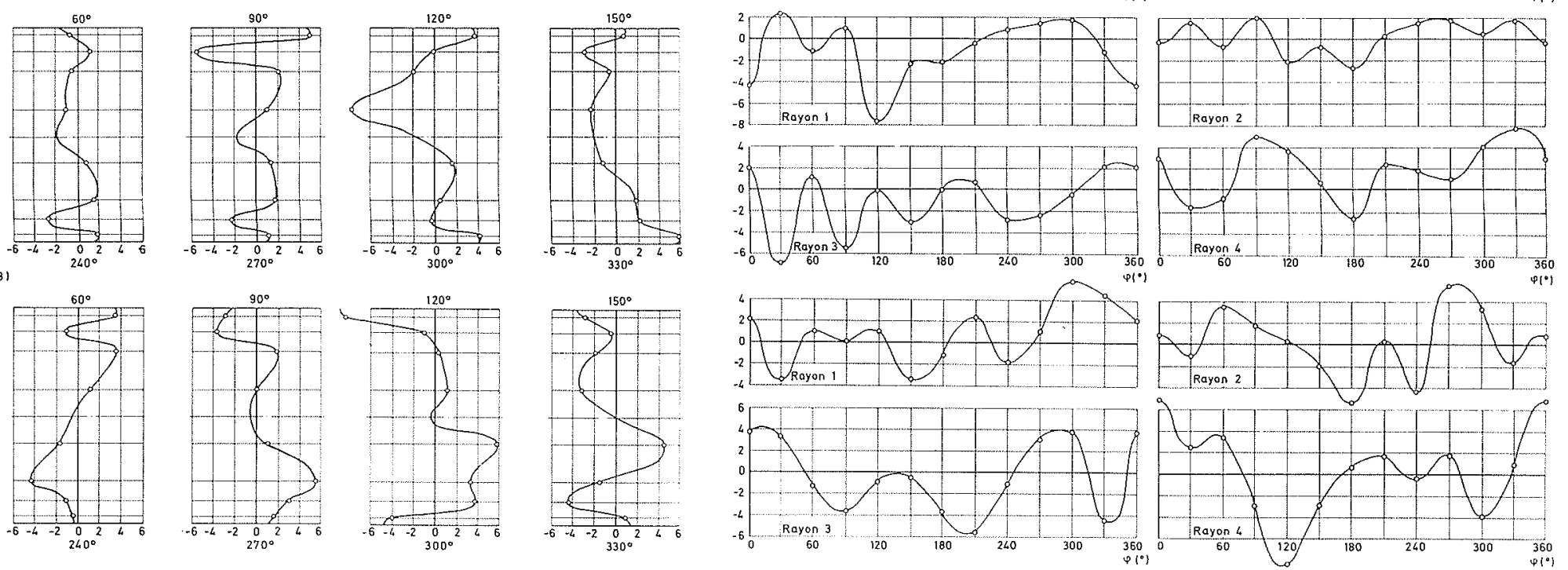

4/ Ecarts de l'énergie totale, par rapport à la valeur moyenne, dans la section de mesure A de la pompe-modèle B en \% 


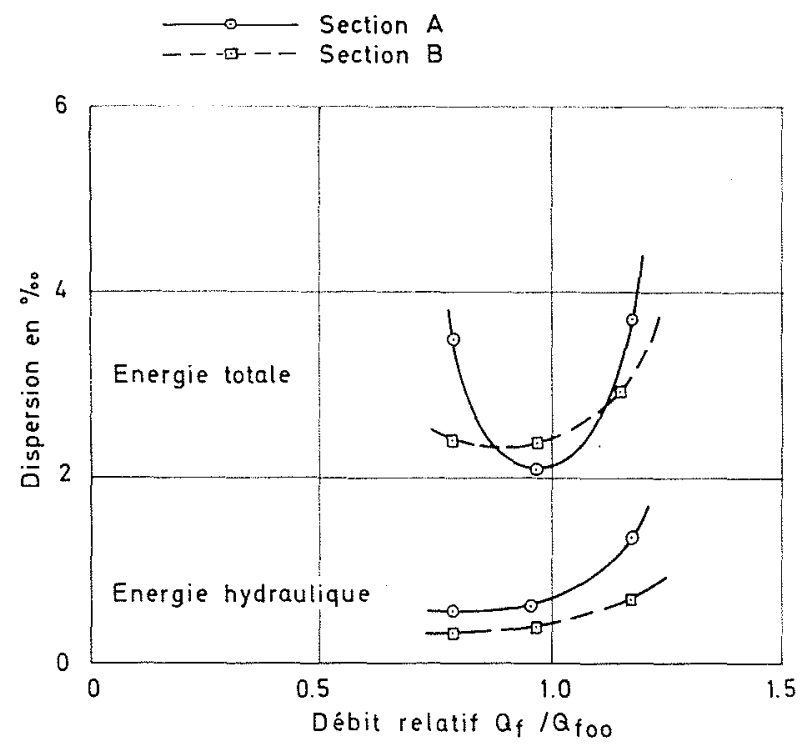

5/Dispersion de l'énergie, suivant le débit relatif, dans la pompemodèle $A$

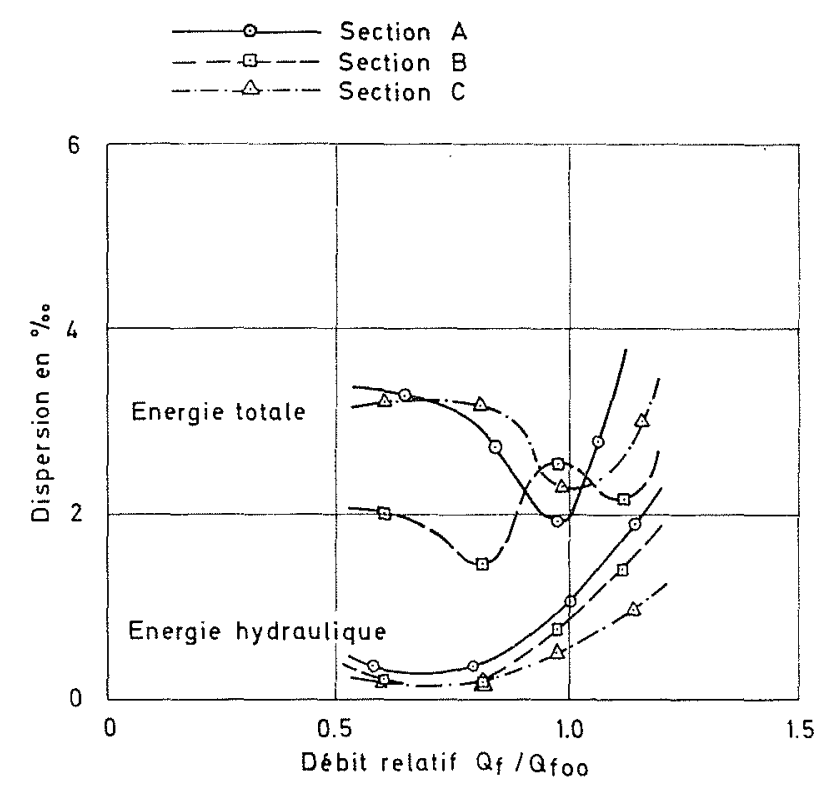

6/ Dispersion de l'énergie, suivant le débit relatif, dans la pompemodèle $B$

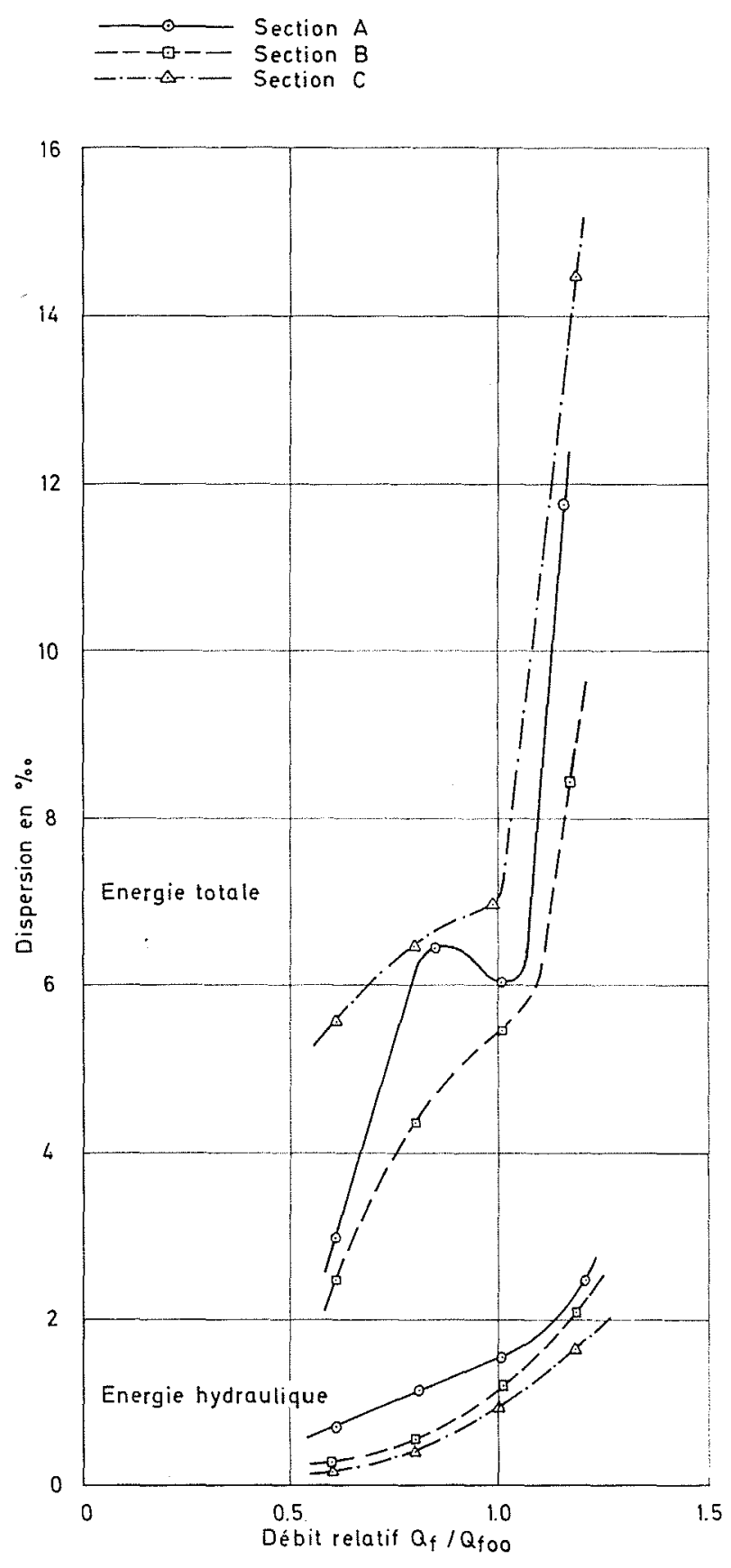

7/ Dispersion de l'énergie, suivant le débit relatif, dans la pompemodèle C

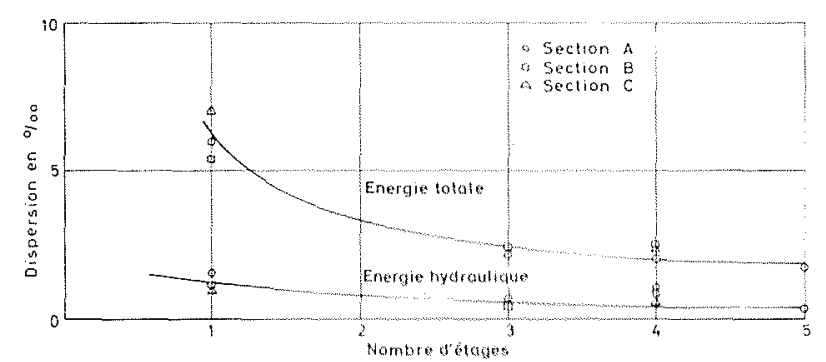

8/Dispersion de l'énergie au point da marcho optimum, suivant le nombre d'átages

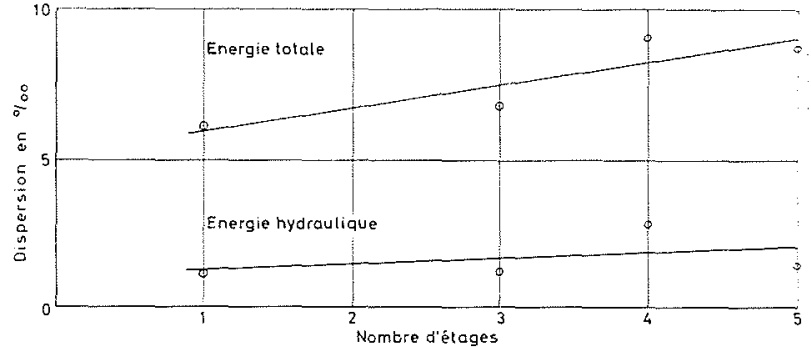

9/ Dispersion de l'énergie au point de marche optimum suivant le nombre d'étages, par rapport à la hauteur de refoulement ou hauteur d'énergie totale d'un étage 


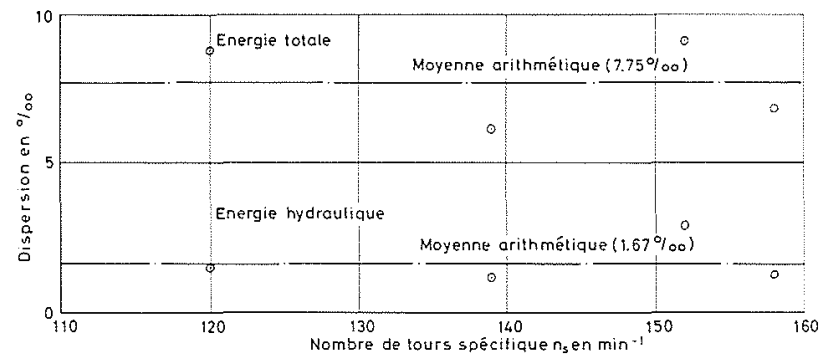

10/Dispersion de l'énergie au point de marche optimum, suivant le nombre de tours spécifique, par rapport à la hauteur de refoulement ou hauteur d'énergie totale d'un étage

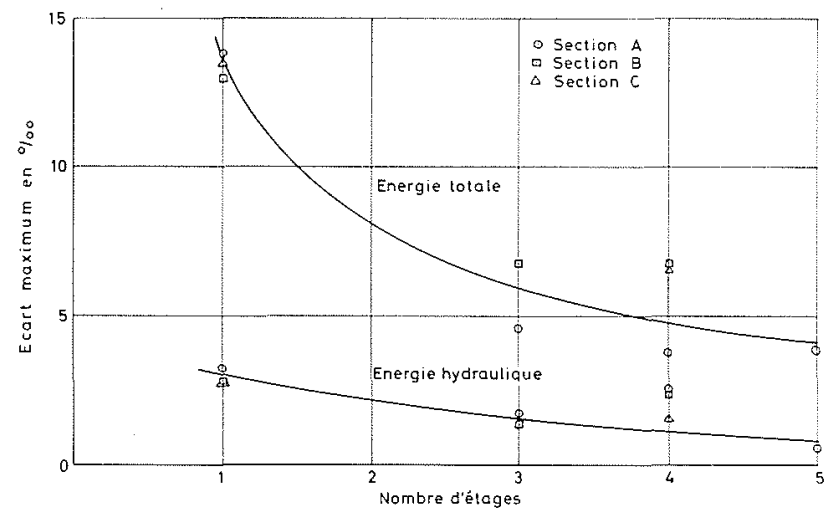

11/Ecart maximum de l'énergie au point de marche optimum, suivant le nombre d'étages

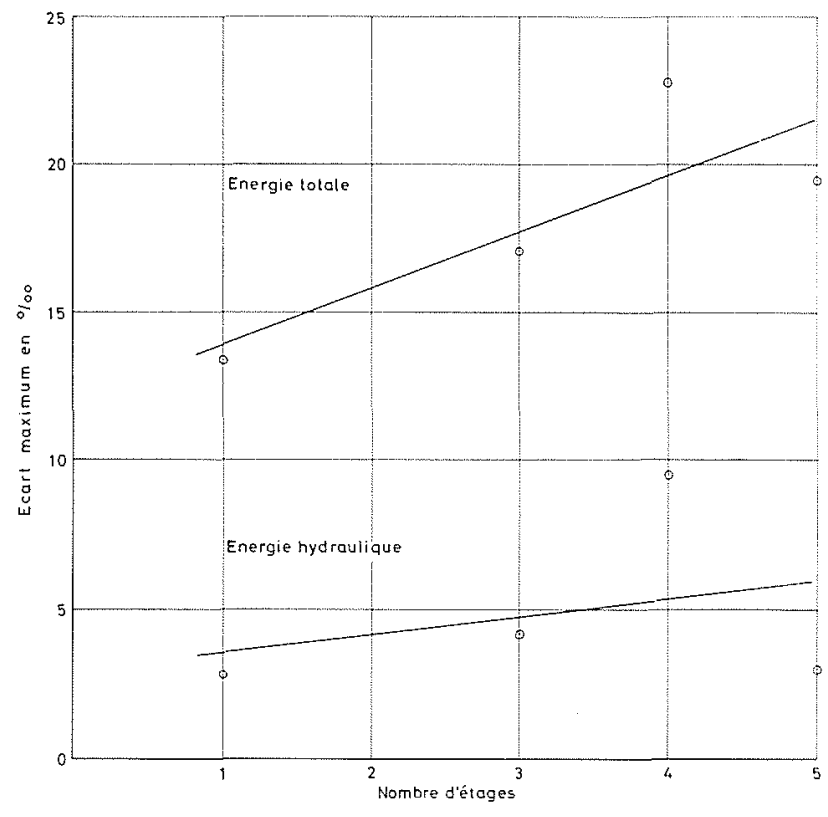

12/Ecart maximum de l'énergie au point de marche optimum, suivant le nombre d'étages, par rapport à la hauteur de refoulement ou hauteur d'énergie totale d'un étage

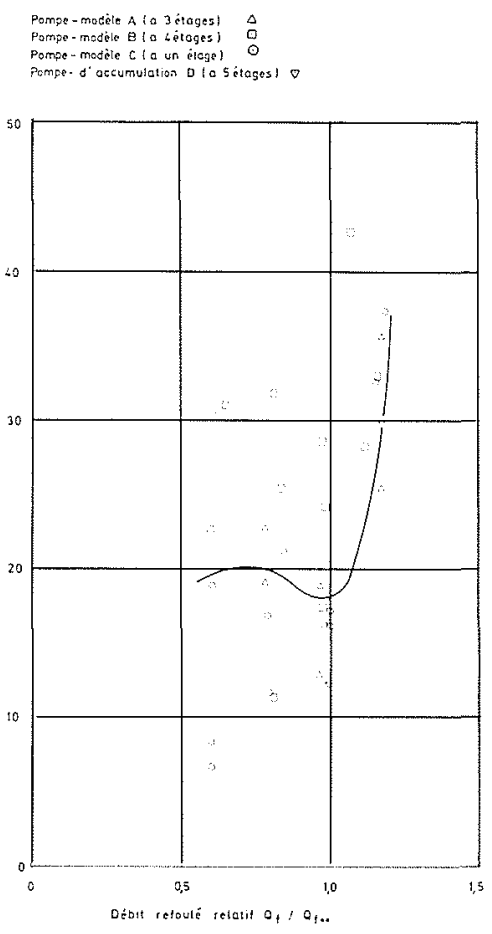

13/Erreur relative de mesure du rendement avec prise unique, suivant le débit refoulé relatif, par rapport à la machine à un seul étage

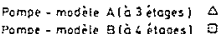

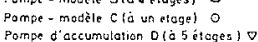

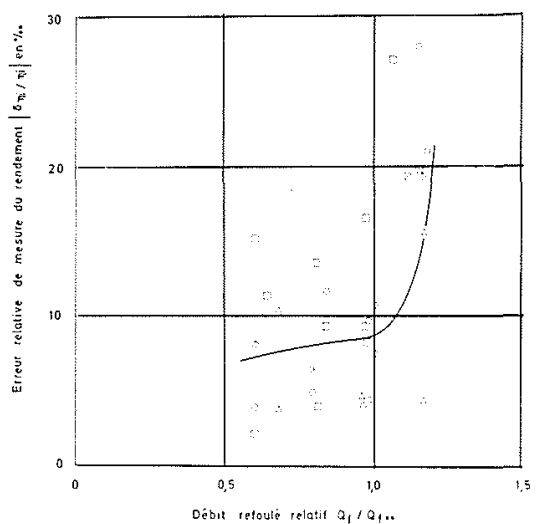

14/Erreur relative de mesure du rendement avec prise unique, à une distance de la paroi de $1 / 7^{\circ}$ du diamètre de la conduite suivant le débit refoulé relatif, par rapport à la machine à un seul étage

\section{Erreurs de mesure}

Au cours des mesures faites en un point isolé d'une section, les valeurs obtenues pour l'énergie hydraulique et pour l'énergie totale sont grevées d'erreurs dont l'importance est déterminée par l'écart local par rapport à la valeur moyenne de l'énergie. En cas d'un choix défa- 
vorable du point de mesure, l'erreur est égale au plus grand écart, par rapport à la valeur moyenne, dans la section en question. Comme le montrent les figures 11 et 12 , qui font ressortir l'influence du nombre d'étages, l'écart le plus important prend la même allure que la dispersion.

L'erreur relative systématique sur le rendement est égale à la somme des erreurs systématiques isolées sur l'énergie hydraulique et sur l'énergie totale, c'est-à-dire à la somme des erreurs les plus grandes sur l'énergie. La figure 13 donne une image des plus importantes erreurs de rendement qui peuvent se produire avec une machine à un seul étage.

Tout au long de la paroi, à une distance égale à $1 / 7^{\mathrm{e}}$ du diamètre de la conduite, les erreurs maximales diminuent d'environ $40 \%$ par rapport aux valeurs correspondant à la section entière. La figure 14 montre les erreurs de rendement les plus grandes qui peuvent se produire avec une machine à un étage, si un point de mesure est disposé le long de la paroi intérieure à une distance égale à $1 / 7^{\mathrm{e}}$ du diamètre de la conduite.

\section{Récapitulation des principaux résultats}

-.. Il n'est pas possible de déterminer sans équivoque aucune, dans la section de mesure, une zone avec répartition optimale de l'énergie, valable pour tous les régimes de marche, tous les genres de machines (nombre d'étages, etc.) et toutes les sections (divers parcours de compensation).

- C'est à l'extérieur de la zone limitée à $1 / 7^{\mathrm{e}}$ de distance de la paroi de la conduite que la répartition de l'énergie est la plus irrégulière. A l'intérieur de cette zone, l'irrégularité la plus forte ne dépasse pas en moyenne $60 \%$ environ des plus hautes valeurs décelées dans la section de mesure tout entière.

- On trouve dans la section de mesure certaines zones dans lequelles la répartition d'énergie est plus favorable que dans celle qui se limite à $1 / 7^{e}$ du diamètre de la conduite, par rapport à la paroi de celle-ci.

- En régime de surcharge, la repartition de l'énergie devient sensiblement plus irrégulière qu'à charge partielle et au régime optimum.

- Une augmentation du parcours de compensation n'a pas d'influence sensible sur l'amélioration de répartition de la température.
- Une erreur éventuelle dans la mesure du rendement d'une pompe est essentiellement une conséquence de la répartition de la température dans la section de mesure. Les irrégularités dans la répartition de l'énergie totale sont en moyenne quatre fois plus importantes que celles de la répartition de l'énergie hydraulique.

- Dans les pompes multicellulaires, les irrégularités, provoquées dans les étages précédant celui de sortie, se propagent sous forme atténuée jusqu'à la sortie de la pompe.

- En évaluant l'erreur probable, dans la mesure du rendement, due à la répartition de la température, il faudra donc tenir compte du nombre d'étages de la pompe examinée.

\section{Bibliographie}

[1] BARBILLON (L.) et POIRSON (A.). - Sur une méthode thermométrique de mesure du rendement des turbines hydrauliques. La Houille Blanche, $1920, \mathrm{~N}^{\circ} 167$, p. $217 / 221$.

[2] BARBILLON (L.) et POIRSON (A.). - Sur la détermination du rendement des turbines hydrauliques. La Houille Blanche, $1921, \mathrm{~N}^{\circ} 172$, p. 161/162.

[3] Poirson (A.) - Méthode thermométrique de mesure du rendement des turbines hydrauliques. Précaution à prendre pour lapplication correcte de cette méthode, La Houille Blanche, $1922, \mathrm{~N}^{\circ} 178$, p. $155 / 157$.

[4] BARBILLON (L.) et POIRSON (A.). - Mesure du rendement des pompes par la méthode thermométrique. La Houille Blanche, $1922, \mathrm{~N}^{\circ} 176$, p. 84.

[5] TRAUPEL (W.). - Thermische Turbomaschinen, Erster Band, Springer Berlin 1958, p. $22 \mathrm{ff}$.

[6] TRAuPEL (W.). - Die Theorie der Stömung durch Radialmaschinen. G. Braun, Karlsruhe 1962, p. 1 ff.

[7] WEBER (P.U.). - Bemerkungen zur thermodynamischen Methode der Wirkungsgradbestimmung von Wasserturbinen und Speicherpumpen, Bulletin $S E V$, vol. 55, 1964, $\mathrm{N}^{\circ} 24$, p. $1199 / 1208$.

[8] WEBER (P.U.), SCHNELLER (H.) et BRUN (J.). - Mesures thermodynamiques du rendement de pompes d'accumulation et de turbines hydrauliques avec l'appareillage "WeberSulzer". Revue Technique Sulzer, $\mathrm{N}^{\circ} 1,1968$, p. 25-32.

[9] VDI. - Regeln für die Durchflussmessung mit genormten Düsen, Blenden und Venturi-Düsen, DIN 1952, $6^{\mathrm{e}}$ édition 1948.

[10] VD1. - Abnahmeversuche an Kreiselpumpen, DIN 1944, octobre 1968.

[11] HENGST (H.) - Einführung in die mathematische Statistik und ihre Anwendung. BI Hochschultaschenbücher, vol. $42 / 42 \mathrm{a}$ Bibliographisches Institut Mannheim/Wien/Zurich 1967. 